\title{
Dietary magnesium intake and risk of hypertension in a Mexican adult population: a cohort study
}

Gerardo G Huitrón-Bravo ${ }^{1}$, Edgar Denova-Gutiérrez ${ }^{2,3 *}$, José de Jesús Garduño-García ${ }^{4,5}$, Juan O Talavera ${ }^{6}$, Benjamín Herreros ${ }^{7}$ and Jorge Salmerón ${ }^{2,8}$

\begin{abstract}
Background: Hypertension is associated with increased risk of heart disease, stroke, kidney failure, premature mortality, and disability. This rising prevalence of hypertension has been linked to insufficient dietary magnesium intake. However, epidemiological evidence supporting this relationship is inconsistent. To investigate whether magnesium intake affects blood pressure, we examined the association between dietary magnesium intake and the incidence of hypertension in apparently healthy Mexican adults participating in the Health Workers Cohort Study.

Methods: A total of 1,378 subjects (77.4\% women and $22.6 \%$ men), participating in the Health Workers Cohort Study, free of hypertension at baseline (systolic blood pressure $<140 \mathrm{mmHg}$ or/and diastolic blood pressure $<90 \mathrm{mmHg}$ ), were prospectively studied. Magnesium intake was evaluated using a semi-quantitative food frequency questionnaire. The longitudinal relationships between dietary magnesium intake and the incidence of hypertension were analyzed with generalized estimation equations.
\end{abstract}

Results: During a median follow-up of 7 years, $16.4 \%$ of women and 31.9\% of men developed incident hypertension. After adjustment for age and sex, we found a trend of decreasing diastolic blood pressure with rising magnesium intake, by tertiles (the coefficients were $-0.75 \mathrm{mmHg}$ [95\% confidence interval (Cl): $-1.77,0.27],-1.27 \mathrm{mmHg}(95 \%$ $\mathrm{Cl}:-2.73,-0.02 ; P$ for trend $=0.01$ ). This inverse association was attenuated after further adjustment for known risk factors. In the fully adjusted model, magnesium intake was inversely associated, although not significantly, with the risk of developing hypertension; subjects in the highest tertile of magnesium intake had a decreased risk for hypertension (odds ratio 0.83 , 95\% Cl: $0.49-1.39$, $P$ for trend $=0.48$ ).

Conclusions: These results do not support the hypothesis that magnesium intake reduces the development of hypertension, although a modest inverse association between magnesium consumption and lower blood pressure cannot be ruled out.

Keywords: Magnesium, Hypertension, Mexican population

\section{Background}

Elevated blood pressure (BP) or hypertension, defined as systolic blood pressure equal to or above $140 \mathrm{mmHg}$ or/and diastolic blood pressure equal to or above $90 \mathrm{mmHg}$, is associated with increased risk of heart disease, stroke, kidney failure, premature mortality, and

\footnotetext{
* Correspondence: edenova03@yahoo.com.mx

${ }^{2}$ Unidad de Investigación Epidemiológica y en Servicios de Salud, Instituto

Mexicano del Seguro Social, Cuernavaca, México

${ }^{3}$ Área Académica de Nutrición, Instituto de Ciencias de la Salud, Universidad Autónoma del Estado de Hidalgo, Pachuca, México

Full list of author information is available at the end of the article
}

disability [1]. Worldwide in 2008, approximately $40 \%$ of adults older than 25 years had been diagnosed with hypertension [1]. In the Americas, 35\% of the adult population suffers from this condition [2]. In Mexico, according to the 2012 National Health and Nutrition Survey [3], 32.4\% of men and 32.3\% of women had hypertension. This rising prevalence of hypertension is linked to population aging and to excess weight and persistent stress exposure on the individual level. Other causes of hypertension include, but are not limited to, tobacco use, high consumption of alcohol, 
sedentary lifestyle, a diet high in sodium, and insufficient intake of minerals like potassium, calcium, and magne$\operatorname{sium}(\mathrm{Mg})[4]$.

Mg plays an important role in cellular processes including vascular tone, cardiac excitability, neuromuscular transmission, and blood pressure [5-7]; additionally, Mg could also decrease BP, since as a calcium antagonist in smooth muscle, it might produce vasorelaxation [8]. Moreover, $\mathrm{Mg}$ diminishes the biological activities of angiotensin II and inhibits norepinephrine release. It has also been reported that plasma rennin activity is inversely associated with serum Mg. Furthermore, it has been shown that variations in extracellular $\mathrm{Mg}$ content modify the production and release of nitric oxide, resulting in the alteration of arterial smooth muscle tone. Finally, it also has been reported that the antioxidant properties of magnesium could attenuate the detrimental effects of oxidative stress on the vasculature, thereby preventing increased vascular tone and contractility $[8,9]$.

Magnesium, a cofactor for hundreds of enzymes, is an essential mineral that is crucial for a numerous of metabolic functions in the human body. The main dietary sources of $\mathrm{Mg}$ are whole grains, legumes, nuts, and green leafy vegetables [10], while processed and refined foods are poor Mg sources [11]. Low Mg consumption is common worldwide (although statistics do not exist for Mexico); in the US, $67 \%$ of women and $64 \%$ of men consume inadequate amounts of $\mathrm{Mg}$ [12]. Insufficient dietary $\mathrm{Mg}$ intake has been related to adverse health outcomes including hypertension [13-15]. However, epidemiological evidence supporting this relationship is inconsistent. For the most part, cross-sectional studies have demonstrated an inverse relationship between dietary $\mathrm{Mg}$ and BP [16-19]. However, prospective cohort studies have also found weak $[20,21]$ or no relationships $[22,23]$ between $\mathrm{Mg}$ and the development of hypertension. Clinical findings on this topic are mixed, including reports that Mg supplementation lowers [24-26] or does not affect [27] BP.

To investigate whether magnesium intake affects BP, we examined the association between dietary magnesium intake and the incidence of hypertension in apparently healthy Mexican adults participating in the Health Workers Cohort Study (HWCS).

\section{Methods}

\section{Study population}

The HWCS is a longitudinal study investigating relationships between lifestyle and health. From March 2004 to April 2006, at baseline examination, it formally enrolled 8,307 medical, administrative, and academic employees and their relatives from three different institutions: the (i) Instituto Mexicano del Seguro Social (IMSS) and (ii) Instituto Nacional de Salud Pública (INSP), both located in Cuernavaca, Morelos State; and workers at the (iii) Universidad Autónoma del Estado de México (UAEM) in Toluca, Mexico State. The participants in this cohort study are middle- to low-income status, residing in urban central Mexico. Study methods and participants' characteristics have been previously described [28,29].

Seven years later, approximately 2,500 subjects of those original HWCS subjects were invited to participate in a follow-up data collection phase, which took place from 2012-2013. Of these, only 1,855 participants attended the second examination and were eligible for the current investigation. Both the participants attending the second examination and no participants in the follow-up study were similar in age, percentage of women, and prevalence of diabetes. Data on sociodemographic, lifestyle, and medical history factors was collected with self-administered questionnaires, and anthropometric measurements and clinical evaluations were performed. For the present analysis of participants in both phases of the HWCS, we excluded individuals with hypertension at baseline $(n=286)$, which we defined as having systolic blood pressure $\geq 140 \mathrm{mmHg}$ or/and diastolic blood pressure $\geq 90 \mathrm{mmHg}$, self-reported history of hypertension, and history of anti-hypertensive treatment; in addition, we eliminated subjects who responded to less than $85 \%$ of questions or did not complete all the diet-related questions $(n=150)$. We also used the standard deviation method to exclude subjects providing extreme caloric intake values $(<600,>7,000 \mathrm{kcal} /$ day $)$ $(n=25)$ and/or extreme magnesium consumption values $(n=16)$ [30]. The final study population included 1,378 men and women without hypertension at baseline and who had provided complete data on Mg intake and other major lifestyle variables.

\section{Ethics statement}

This study was planned and performed according to the guidelines of the Declaration of Helsinki. All participating institutions' research ethics committees (Comité de Ética en Investigación, Instituto Mexicano del Seguro Social (No. 12CEI 09006 14); Comité de ética en Investigación, Instituto Nacional de Salud Pública (No. 13CEI 17007 36); Comité de Ética, Centro de Investigación en Ciencias Médicas (No. 1233008X0236)) approved the study protocol and informed consent forms. Written informed consent was obtained from all participants.

\section{Assessment of magnesium intake}

In the HWCS, a validated 116-item semi-quantitative food frequency questionnaire (FFQ) was used to collect dietary information [31]. On the FFQ, a common unit or portion size for each food was specified and the participants were asked how often they had consumed that amount on average during the previous year. Ten possible responses 
ranging from "never" to "six or more times per day" were recorded. Magnesium and other nutrient intakes were computed by multiplying the frequency of consumption of each unit of food from the FFQ by the magnesium and nutrient content of the specified portion size. Composition values for dietary magnesium and other nutrients were obtained from a Mexican database of food contents [32]. Information about use of specific brand and type of multivitamins was collected by asking current users about weekly number of multivitamins taken. Total magnesium represented the sum of magnesium intake from dietary and supplemental sources. Each nutrient was adjusted for total energy using the residual method [33]. Finally, in order to compute energy consumption, the daily frequency of consumption of each food was multiplied by the food's energy content.

\section{Assessment of anthropometric measures}

Body weight was measured with a previously calibrated electronic scale (model BC-533; Tanita, Tokyo, Japan), with participants wearing minimal clothing and no shoes. Height was measured using a conventional stadiometer (SECA brand) on barefoot subjects standing with their shoulders in a normal position; the measurements were taken with a tape in a horizontal plane perpendicular to the vertical scale, touching the top of the head at the moment of inspiration. Body mass index (BMI) was computed as a ratio of weight in kilograms divided by the height in meters squared.

\section{Assessment of hypertension}

Subjects' blood pressure was measured twice during each study visit by a trained nurse using an automatic monitor. The first measurement was taken after $5 \mathrm{~min}$ of rest, while participants were sitting with the dominant arm supported at heart level. The second measurement was taken in the same way, 5 min after the first.

Incident hypertension was considered present if the subject had received treatment for high blood pressure and/or physician diagnosis (systolic blood pressure equal to or above $140 \mathrm{mmHg}$ or/and diastolic blood pressure equal to or above $90 \mathrm{mmHg}$ ) in our cohort study.

All measurement procedures were performed by nurses trained to use standardized procedures (reproducibility was evaluated, resulting in concordance coefficients between 0.83 and 0.90$)$.

\section{Assessment of other variables}

Information on participants' sociodemographic characteristics (e.g., age, sex, and education), medical history, and lifestyle, including alcohol and tobacco consumption, were collected using a self-administered questionnaire.
Physical activity level was determined using a survey in which participants reported the time they spent each week on activities such as running and walking during a typical week in the previous year. Each activity was given a value in metabolic equivalent tasks (METs) and total METs per week was computed.

\section{Statistical analysis}

A descriptive analysis of the main variables of interest (age, BMI, waist circumference, physical activity, total energy intake, etc.) across tertiles of total dietary magnesium intake was performed. Analysis of variance (ANOVA) was used to evaluate mean differences across tertiles of total dietary magnesium intake. The chi-square test was used to determine differences in the distribution of qualitative variables across tertiles of total dietary magnesium intake.

Longitudinal coefficients were calculated between magnesium intake (tertiles) and systolic and diastolic blood pressure using the generalized estimating equations (GEE) technique [34]. This technique tests the relationship between systolic and diastolic blood pressure and magnesium intake at a specific time point, resulting in a standardized regression coefficient which can be interpreted as a longitudinal coefficient. This method corrects for time-dependent covariates, like year of measurement and biological age, and time-independent covariates like sex. We also fitted GEE models to study the association between magnesium intake and incident hypertension. This approach enabled us to describe time-dependent relationships between magnesium intake and hypertension status (yes or no), after adjusting for potential confounders. In each GEE model, we chose "Logit" as the link function because hypertension status was a binary dependent variable.

To compute regression coefficients and odds ratios (ORs), we fitted four multivariate models. The first model was adjusted for age and sex. The second model added smoking (never, past, and current), physical activity (METs per week), alcohol intake (non-drinker/drinker), postmenopausal hormone use (yes/no), and use of multivitamin supplements (yes/no). The third model included additional adjustments for dietary intake of saturated fat (g/day), cholesterol (mg/day), sodium (mg/day), dietary glycemic load (quintiles), fiber (g/day), and total energy intake (kcal/day). The final model additionally controlled for body mass index $\left(\mathrm{kg} / \mathrm{m}^{2}\right)$, history of diabetes mellitus (yes/no), and family history of hypertension. Tests of linear trend across increasing tertiles of magnesium intake were conducted by assigning the means of intakes in tertiles, treated as a continuous variable.

All $P$ values presented are two sided; $P<0.05$ was considered statistically significant. The statistical analyses were performed using the STATA statistical software 
package, version 11.0 for Windows (Stata Corp. LP: College Station, TX).

\section{Results}

The present study includes $77.4 \%$ of women and $22.6 \%$ of men aged 20-87 years and free of hypertension at baseline. During the approximately 7 years between the baseline and follow-up assessments, 16.4\% of women and $31.9 \%$ of men became hypertensive. The median intake of $\mathrm{Mg}$ was $308 \mathrm{mg} /$ day. Baseline characteristics (sociodemographics, body composition, clinical parameters, and dietary information, standardized by age and sex), are shown in Table 1 for the sample used in the incident hypertension analysis. The highest tertile of magnesium intake had a higher proportion of men, and subjects in that tertile were slightly older and were less likely to be current smokers, to exercise, or to take multivitamins or postmenopausal hormones than those in the lowest tertile of $\mathrm{Mg}$ consumption. $\mathrm{Mg}$ intake was also inversely associated with intake of fat and cholesterol and positively associated with the consumption of fiber and glycemic load (Table 1).

Table 2 lists the results of a multivariate GEE analysis evaluating the effects of $\mathrm{Mg}$ intake on systolic and diastolic

Table 1 Baseline characteristics across categories of magnesium intake in adults of the Health Worker Cohort Study

\begin{tabular}{|c|c|c|c|c|}
\hline \multirow[t]{2}{*}{ Characteristics } & \multicolumn{3}{|l|}{ Magnesium intake } & \multirow[t]{2}{*}{$P$ value } \\
\hline & $\mathrm{T1}(n=459)$ & T2 $(n=459)$ & T3 $(n=460)$ & \\
\hline Age (years) ${ }^{\mathrm{b}}$ & $40.7(15.1)$ & $41.7(14.7)$ & $41.9(15.2)$ & 0.451 \\
\hline Sex (\% women) & 76.0 & 76.5 & 73.2 & 0.473 \\
\hline Body mass index $(\mathrm{kg} / \mathrm{m} 2)^{\mathrm{b}}$ & $25.4(4.1)$ & $25.5(4.4)$ & $25.5(4.4)$ & 0.583 \\
\hline Waist circumference $(\mathrm{cm})^{\mathrm{b}}$ & $87.7(11.1)$ & $88.1(12.7)$ & $88.2(12.6)$ & 0.433 \\
\hline \multicolumn{5}{|l|}{ Systolic blood pressure (mmHg) } \\
\hline$<110^{c}$ & 35.7 & 32.9 & 35.8 & \multirow[t]{4}{*}{0.748} \\
\hline $110-119^{c}$ & 35.5 & 34.4 & 33.0 & \\
\hline $120-129^{c}$ & 18.8 & 22.7 & 22.2 & \\
\hline $130-139^{c}$ & 10.0 & 10.0 & 9.0 & \\
\hline \multicolumn{5}{|l|}{ Diastolic blood pressure $(\mathrm{mmHg})$} \\
\hline$<65^{c}$ & 31.6 & 27.1 & 28.3 & \multirow[t]{4}{*}{0.477} \\
\hline $65-74^{c}$ & 39.7 & 44.4 & 39.6 & \\
\hline $75-84^{c}$ & 26.7 & 25.9 & 30.0 & \\
\hline $85-89^{c}$ & 2.0 & 2.6 & 2.1 & \\
\hline Current smoker $^{\mathrm{c}}$ & 16.2 & 16.2 & 14.7 & 0.818 \\
\hline Alcohol consumption $(\mathrm{mg} / \text { day })^{\mathrm{b}}$ & $3.9(1.3)$ & $4.2(1.3)$ & $4.1(1.3)$ & 0.103 \\
\hline Physical activity (METs/week) ${ }^{b}$ & $146.1(66.1)$ & $145.6(58.9)$ & $145.6(62.1)$ & 0.602 \\
\hline Postmenopausal hormones (current user) ${ }^{c}$ & 29.9 & 23.9 & 23.0 & 0.285 \\
\hline Multivitamin use (current user) ${ }^{c}$ & 37.0 & 37.7 & 34.2 & 0.505 \\
\hline History of hyperlipidemia ${ }^{c}$ & 15.0 & 13.5 & 15.8 & 0.583 \\
\hline Diabetes mellitus ${ }^{c}$ & 6.0 & 4.5 & 4.0 & 0.358 \\
\hline Total caloric intake (kcal/day) ${ }^{\mathrm{b}}$ & $2,143(148)$ & $2,135(144)$ & $2,138(147)$ & 0.675 \\
\hline Glycemic load ${ }^{\mathrm{b}}$ & $201(16.2)$ & $303(10.1)$ & $464(11.0)$ & $<0.001$ \\
\hline Total fat intake $(\mathrm{g} / \text { day })^{\mathrm{b}}$ & $74.0(32.6)$ & $52.1(21.6)$ & $46.9(21.4)$ & $<0.001$ \\
\hline Total protein intake $(\mathrm{g} / \text { day })^{\mathrm{b}}$ & $95.2(38.8)$ & $69.4(25.5)$ & $63.4(22.9)$ & $<0.001$ \\
\hline Total cholesterol (mg/day) & $297.7(93.2)$ & $219.5(63.7)$ & $201.1(64.6)$ & $<0.001$ \\
\hline Sodium intake $(\mathrm{mg} / \text { day })^{\mathrm{b}}$ & $2497(850)$ & $1783(581)$ & $1615(589)$ & $<0.001$ \\
\hline Fiber intake $(g / \text { day })^{b}$ & $14.4(6.1)$ & $16.6(6.3)$ & $19.2(6.8)$ & $<0.001$ \\
\hline Magnesium intake $(\mathrm{mg} / \text { day })^{d}$ & $336.2(223.2-381.1)$ & $359.3(266.9-419.3)$ & $420.6(322.4-522.2)$ & $<0.001$ \\
\hline
\end{tabular}

All variables have been analyzed after adjustment for age (5-year categories) and sex. All covariate values are listed according to the tertile of total magnesium intake. ${ }^{a}$ ANOVA test was used for quantitative variables; $X^{2}$ test was used for qualitative variables.

balues are presented as means and SD (in parentheses).

${ }^{\complement}$ Values are presented as proportions.

${ }^{d}$ Values are presented as means and interquartile range (in parentheses). 


\begin{tabular}{|c|c|c|c|c|}
\hline \multirow[t]{3}{*}{ Outcomes } & \multicolumn{3}{|c|}{ Total magnesium intake (tertiles) } & \multirow[t]{3}{*}{$P$ value $^{\mathrm{e}}$} \\
\hline & $\overline{\mathrm{T} 1}$ & T2 & T3 & \\
\hline & $\beta(95 \% \mathrm{Cl})$ & $\beta(95 \% \mathrm{Cl})$ & $\beta(95 \% \mathrm{Cl})$ & \\
\hline Diastolic blood pressure $(\mathrm{mmHg})^{\mathrm{a}}$ & 0.0 & $-0.75(-1.77,0.27)$ & $-1.27(-2.73,-0.02)$ & 0.01 \\
\hline Systolic blood pressure $(\mathrm{mmHg})^{\mathrm{a}}$ & 0.0 & $-0.23(-1.62,1.07)$ & $-0.70(-2.04,0.65)$ & 0.31 \\
\hline Diastolic blood pressure $(\mathrm{mmHg})^{\mathrm{b}}$ & 0.0 & $-0.72(-1.71,1.34)$ & $-1.11(-4.38,0.03)$ & 0.05 \\
\hline Systolic blood pressure $(\mathrm{mmHg})^{\mathrm{b}}$ & 0.0 & $-0.20(-1.57,1.18)$ & $-0.47(-1.86,0.92)$ & 0.50 \\
\hline Diastolic blood pressure $(\mathrm{mmHg})^{c}$ & 0.0 & $-0.68(-1.76,0.41)$ & $-1.16(-2.40,0.07)$ & 0.06 \\
\hline Systolic blood pressure $(\mathrm{mmHg})^{c}$ & 0.0 & $-0.08(-1.50,1.35)$ & $-0.40(-2.03,1.24)$ & 0.64 \\
\hline Diastolic blood pressure $(\mathrm{mmHg})^{d}$ & 0.0 & $-0.60(-1.65,0.45)$ & $-1.12(-2.31,0.08)$ & 0.06 \\
\hline Systolic blood pressure $(\mathrm{mmHg})^{d}$ & 0.0 & $-0.01(-1.35,1.35)$ & $-0.35(-1.92,1.21)$ & 0.66 \\
\hline
\end{tabular}

${ }^{a}$ Adjusted for age (years), sex, and time.

${ }^{\mathrm{b}}$ Further adjusted for physical activity (METs/week), postmenopausal hormone use (yes/no), multivitamin use (yes/no), alcohol consumption (non-drinker/drinker), smoking (never, past, and current).

${ }^{c}$ Multivariate model additionally adjusted for dietary intakes of saturated fat (g/day), cholesterol (mg/day), glycemic load (quintiles), sodium intake (mg/day), fiber (g/day), and total energy (kcal/day).

${ }^{d}$ A full model with additional adjustment for body mass index $\left(\mathrm{kg} / \mathrm{m}^{2}\right)$, history of diabetes, and family history of hypertension.

'Test for linear trend.

blood pressure. In the first model, adjusted for age and sex, we found an inverse and significant relationship between magnesium intake and diastolic blood pressure, with $\beta$ [95\% confidence interval $(\mathrm{CI})]$ comparing the top versus the bottom tertiles of $-1.27 \mathrm{mmHg}(-2.73,-0.02 ; P$ for linear trend $=0.01$ ). A weak negative coefficient was observed between magnesium intake and systolic blood pressure, but this relationship was not statistically significant $(\beta=-0.70 ; 95 \% \mathrm{CI}=-2.04,0.65 ; P$ for linear trend $=0.31$ ). Comparing the highest and lowest tertiles of magnesium consumption, in the fourth model (adjusted for age, sex, physical activity, postmenopausal hormone use, multivitamin use, alcohol consumption, smoking, body mass index, history of diabetes, and dietary intake of saturated fat, cholesterol, glycemic load, sodium intake, fiber, and total energy), the inverse relationship between magnesium intake and diastolic blood pressure was attenuated and was not statistically significant $(\beta=-1.12 ; 95 \% \mathrm{CI}=$ $-2.31,0.08 ; P$ for linear trend $=0.06$ ). Finally, in the full model (which also included body mass index, history of diabetes, and family history of hypertension), we found an inverse, but not significant relationship between magnesium intake and systolic blood pressure $(\beta=-0.35 ; 95 \%$ $\mathrm{CI}=-1.92,1.21 ; P$ for linear trend $=0.66$ ).

Table 3 shows the ORs and 95\% CIs of multiple GEE analysis for incident hypertension. The multiple GEE models reveal that magnesium intake was inversely associated with risk of hypertension, but not significantly $(P$ for linear trend $=0.48$ ). After adjustment for major confounders, we observed a negative association with an OR of 0.83 (95\% $\mathrm{CI}=0.49-1.39)$ when we compared the subjects with the lowest level of magnesium intake (T1) and subjects in the highest level of magnesium intake (T3).

\section{Discussion}

In the present prospective cohort study with an approximately 7-year period of follow-up, we found a modestly decreasing trend of diastolic blood pressure according to higher amount of magnesium intake. However, our results suggest no significant association between dietary magnesium intake and the subsequent development of hypertension in this adult Mexican population.

The relationship between magnesium and blood pressure has been studied for decades. Many observational studies have found an inverse association between magnesium consumption and blood pressure, but this relationship is not conclusively supported. Numerous cross-sectional studies have linked dietary magnesium intake to lower prevalence of hypertension $[17,18]$, with most, but not all, finding negative associations between magnesium intake and blood pressure, although the cross-sectional association appears to be consistent, given the characteristics of this study design that finding does not necessarily imply a causal relationship.

Evidence from prospective cohort studies has been inconsistent [15,20,21,35-37]. For example, in the US, in a 10-year follow-up study, Song et al. [20] found that subjects in the highest quintile of magnesium intake had a decreased risk for hypertension (relative risk $=0.91$, 95\% CI: $0.83-0.99, P$ for linear trend $=0.002$ ) compared with subjects in the lowest quintile. In contrast, our findings are consistent with those from the Health Professionals Follow-up Study conducted by Ascherio et al. [36] and the Atherosclerosis Risk in Communities Study done by Peacock et al. [21], which found weak and nonsignificant associations between magnesium consumption and hypertension. In general, available evidence from 
Table 3 Odds ratios of multiple GEE analysis for incident hypertension after adjusting for potential confounding variables

\begin{tabular}{|c|c|c|c|c|}
\hline \multirow[t]{3}{*}{ Outcomes } & \multicolumn{3}{|c|}{ Total magnesium intake (tertiles) } & \multirow[t]{3}{*}{$P$ value $^{\mathrm{e}}$} \\
\hline & T1 & T2 & T3 & \\
\hline & OR $(95 \% \mathrm{Cl})$ & OR $(95 \% \mathrm{Cl})$ & OR $(95 \% \mathrm{Cl})$ & \\
\hline Hypertension (model 1) ${ }^{\mathrm{a}}$ & 1.0 & $0.91(0.60-1.38)$ & $0.85(0.55-1.30)$ & 0.46 \\
\hline Hypertension (model 2) ${ }^{b}$ & 1.0 & $1.0(0.65-1.58)$ & $0.93(0.51-1.44)$ & 0.76 \\
\hline Hypertension (model 3) ${ }^{c}$ & 1.0 & $1.0(0.64-1.58)$ & $0.86(0.51-1.44)$ & 0.57 \\
\hline Hypertension (model 4) ${ }^{d}$ & 1.0 & $0.99(0.63-1.57)$ & $0.83(0.49-1.39)$ & 0.48 \\
\hline
\end{tabular}

Hypertension: systolic blood pressure $\geq 140 \mathrm{mmHg}$ or/and diastolic blood pressure $\geq 90 \mathrm{mmHg}$.

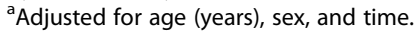

${ }^{b}$ Further adjusted for physical activity (METs/week), postmenopausal hormone use (yes/no), multivitamin use (yes/no), alcohol consumption (non-drinker/drinker), smoking (never, past and current).

${ }^{c}$ Multivariate model additionally adjusted for dietary intakes of saturated fat (g/day), cholesterol (mg/day), glycemic load (quintiles), sodium intake (mg/day),

fiber ( $\mathrm{g} /$ day), and total energy (kcal/day).

${ }^{\mathrm{d}}$ A full model with additional adjustment for body mass index $\left(\mathrm{kg} / \mathrm{m}^{2}\right)$, history of diabetes, and family history of hypertension.

'Test for linear trend.

prospective studies does not demonstrate that magnesium intake plays a primary role in the prevention of hypertension, although the possibility that high magnesium intake has a minor effect in reducing blood pressure in normotensive subjects should not be ruled out. Although some cohort studies have found lack of association between dietary magnesium intake and the risk of hypertension, there has been recent interest in magnesium therapy as a method to reduce blood pressure. However, clinical evidence for blood pressure reduction with magnesium supplementation remains equally unclear [24-26]. A recent meta-analysis of 22 clinical trials suggests a negative association between magnesium supplementation and diastolic and systolic blood pressure; these trials found an average reduction of $2-3 \mathrm{mmHg}$ for diastolic blood pressure and 3-4 mmHg for systolic blood pressure [38].

In order to understand the lack of association between dietary magnesium intake and the subsequent development of hypertension in this adult Mexican population, some limitations of the present cohort should be considered. Some of these limitations relate to the measurement of nutrient consumption and effects. First, errors in the measurement of self-reported dietary intake are inevitable. However, because the data were prospectively collected, the misclassification of dietary intake was likely to be nondifferential and could have led to an underestimation of the association between dietary magnesium intake and the risk of hypertension in our study. Additionally, these errors are unlikely to affect our results, since the FFQ that we used in this study has been validated [31] as reasonably reflecting long-term dietary intake. Second, there is a high degree of multicolinearity among magnesium and several other nutrients that makes it difficult to completely separate the independent effects of magnesium from those of nutrients like fiber, sodium, potassium, folate, and calcium. Third, in our cohort, we observed a relatively narrow range of magnesium intake, which could limit our ability detect a moderate association between magnesium intake and blood pressure. Fourth, while the prospective design of our study reduces the possibility of recall or selection bias, the low response rate at follow-up and the small sample size may have influenced our results, which were not statistically significant.

Other study limitations relate to possible confounding of data. First, the participants might have changed their diets after developing disease, leading to a conservative estimate of the relationship between magnesium and blood pressure. However, this is unlikely since the model that excluded participants who had histories hypertension or dyslipidemia generated null associations similar to other models. Second, we did not separately compare the effects of dietary magnesium and supplementary magnesium, but since subjects' intake of magnesium from supplements was minor compared with dietary intake, this was not likely to have influenced the results. Finally, while we adjusted for potential confounding factors, the possibility of residual or unmeasured confounds still remains. In addition, the participants in this cohort study are adults from a specific segment of the Mexican population: working class, seemingly healthy individuals. While they cannot be considered representative of the Mexican adult population as a whole, they may be considered representative of middle- to low-income adults residing in the urban areas of central Mexico.

\section{Conclusions}

In conclusion, these results do not support the hypothesis that magnesium intake reduces the development of hypertension, although a modest inverse association with blood pressure cannot be ruled out.

\section{Abbreviations}

BP: Blood pressure; Mg: Magnesium; HWCS: Health Workers Cohort Study; FFQ: Food frequency questionnaire; BMI: Body mass index; METs: Metabolic 
equivalent tasks; ANOVA: Analysis of variance; GEE: Generalized estimating equations; OR: Odds ratio; 95\% Cl: 95\% confidence interval.

\section{Competing interests}

Our manuscript is not a duplicate of any previously submitted paper for publication, and all authors take responsibility for this manuscript as well as approve its final form. The authors declare that they have no competing interests.

\section{Authors' contributions}

GGHB was the main author and responsible of the article. EDG analyzed the data, wrote the paper, and had responsibility for its final content. JOT, JJGG, and $\mathrm{BH}$ assisted with writing and had responsibility for the final content. JS participated in research design and execution, as well as in writing the manuscript. All authors read and approved the final manuscript.

\section{Acknowledgements}

Financial support for this study was provided by the Intituto Mexicano del Seguro Social (IMSS), the Consejo Nacional de Ciencia y Tecnología (CONACyT) grant (No. 87783), and the Universidad Autónoma del Estado de México (UAEMex) grant (No. 2682/2008).

\section{Author details}

${ }^{1}$ Facultad de Medicina and Centro de Investigación en Ciencias Médicas, Universidad Autónoma del Estado de México, Toluca, México. ${ }^{2}$ Unidad de Investigación Epidemiológica y en Servicios de Salud, Instituto Mexicano del Seguro Social, Cuernavaca, México. ${ }^{3}$ Área Académica de Nutrición, Instituto de Ciencias de la Salud, Universidad Autónoma del Estado de Hidalgo, Pachuca, México. ${ }^{4}$ Instituto Mexicano del Seguro Social, Hospital General Regional 251, Toluca, México. ${ }^{5}$ Centro de Investigación en Ciencias Médicas, Universidad Autónoma del Estado de México, Toluca, México. ${ }^{6}$ Unidad de Investigación Médica en Epidemiologia Clínica, Centro Médico Nacional Siglo XXI, Instituto Mexicano del Seguro Social, México, DF, México. Instituto de Ética Clínica Francisco Valles, Universidad Europea de Madrid, Madrid, España. ${ }^{8}$ Centro de Investigación en Salud Poblacional, Instituto Nacional de Salud Pública, Cuernavaca, Morelos, México.

Received: 2 September 2014 Accepted: 11 December 2014 Published: 17 February 2015

\section{References}

1. Lim SS, Vos T, Flaxman AD, Danaei G, Shibuya K, Adair-Rohani H, et al. A comparative risk assessment of burden of disease and injury attributable to 67 risk factors and risk factor clusters in 21 regions, 1990-2010: a systematic analysis for the Global Burden of Disease Study 2010. Lancet. 2012:380:2224-60

2. World Health Organization. Global Status Report on Noncommunicable Diseases 2010. Geneva: World Health Organization; 2011.

3. Gutiérrez JP, Rivera-Dommarco J, Shamah-Levy T, Villalpando-Hernández S, Franco A, Cuevas-Nasu L, et al. Encuesta Nacional de Salud y Nutrición 2012. Resultados Nacionales. Cuernavaca, México: Instituto Nacional de Salud Pública; 2012

4. Lawes CM, Vander Hoorn S, Rodgers A, The International Society of Hypertension. Global burden of blood-pressure-related disease, 2001. Lancet. 2008:371:1513-8.

5. Champagne CM. Magnesium in hypertension, cardiovascular disease, metabolic syndrome, and other conditions: a review. Nutr Clin Prac. 2008:23:142-51.

6. Barbagallo M, Dominguez L, Galioto A, Ferlisi A, Cani C, Malfa L, et al. Role of magnesium in insulin action, diabetes and cardio-metabolic syndrome $\mathrm{X}$. Mol Aspects Med. 2003;24:39-52.

7. Ueshima K. Magnesium and ischemic heart disease: a review of epidemiological, experimental and clinical evidences. Magnes Rev. 2005;18:275-84.

8. Bo S, Pisu E. Role of magnesium in cardiovascular disease prevention, insulin sensitivity and diabetes. Curr Opin Lipidol. 2008;19:50-6.

9. Rodríguez-Moran M, Guerrero-Romero F. Hypomagnesemia and prehypertension in otherwise healthy individuals. Eur J Intern Med. 2014;25:128-31.

10. He K, Song Y, Belin RJ, Chen Y. Magnesium intake and the metabolic syndrome: epidemiologic evidence to date. JCMS. 2006;1:351-5.
11. Ford ES, Mokdad AH. Dietary magnesium intake in a national sample of U.S. adults. J Nutr. 2003;133:2879-82.

12. Moshfegh A, Goldman J, Cleveland L. What We Eat in America, NHANES 2001-2002: Usual Nutrient Intakes from Food Compared to Dietary References Intake. Washington, DC: Agricultural Research Service, US Department of Agriculture; 2005. p. 1-56.

13. Song Y, Manson JE, Cook NR, Albert CM, Buring JE, Liu S. Dietary magnesium intake and risk of cardiovascular disease among women. Am J Cardiol. 2005;96:1135-41.

14. Larson SG, Virtanen MJ, Mars M, Mannisto S, Pietinen P, Albanes D, et al. Magnesium, calcium, potassium, and sodium intakes and risk of stroke in male smokers. Arch Inter Med. 2008;168:459-65.

15. Joffres MR, Reed DM, Yano K. Relationship of magnesium intake and other dietary factors to blood pressure: the Honolulu heart study. Am J Clin Nutr. 1987:45:469-75.

16. Sugiyama T, Xie D, Graham-Maar RC, Inoue K, Kobayashi Y, Stettler N Dietary and lifestyle factors associated with blood pressure among U.S. adolescents. J Adoles Health. 2007:40:166-72.

17. Mizushima S, Cappuccio FP, Nichols R, Elliott P. Dietary magnesium intake and blood pressure: a qualitative overview of the observational studies. J Hum Hypertens. 1998;12:447-53.

18. McKeown NM, Jacques PF, Zhang XL, Juan W, Sahyoun NR. Dietary magnesium intake is related to metabolic syndrome in older Americans. Eur J Nutr. 2008:47:210-6.

19. Chen Y, Factor-Litvak P, Howe GR, Parvez F, Ahsan H. Nutritional influence on risk of high blood pressure in Bangladesh: a population-based cross-sectional study. Am J Clin Nutr. 2006;84:1224-32.

20. Song Y, Sesso HD, Manson JE, Cook NR, Buring JE, Liu S. Dietary magnesium intake and risk of incident hypertension among middle-aged and older US women in a 10-year follow-up study. Am J Cardiol. 2006;98:1616-21.

21. Peacock JM, Folsom AR, Arnett DK, Eckfeldt JH, Szklo M. Relationship of serum and dietary magnesium to incident hypertension: the Atherosclerosis Risk in Communities (ARIC) Study. Ann Epidemiol. 1999;9:159-65.

22. Khan AM, Sullivan L, McCabe E, Levy D, Vasan RS, Wang TJ. Lack of association between serum magnesium and the risk of hypertension and cardiovascular disease. Am Heart J. 2010:160:715-20.

23. Joosten MM, Gansevoort RT, Mukamal KJ, Kootstra-Ros JE, Feskens EJ, Geleijnse JM, et al. Urinary magnesium excretion and risk of hypertension: the prevention of renal and vascular end-stage disease study. Hypertension. 2013:61:1161-7.

24. Wexler R, Aukerman G. Nonpharmacologic strategies for managing hypertension. Am Fam Physician. 2006;73:1953-6.

25. Guerrero-Romero F, Rodríguez-Moran M. The effect of lowering blood pressure by magnesium supplementation in diabetic hypertensive adults with low serum magnesium levels: a randomized, double-blind, placebocontrolled clinical trial. J Hum Hypertens. 2009;23:245-51.

26. Yokota K, Kato M, Lister F, Ii H, Hayakawa T, Kikuta T, et al. Clinical efficacy of magnesium supplementation in patients with type 2 diabetes. J Am Col Nutr. 2004:23:506S-9.

27. Zemel PC, Zemel MB, Urberg M, Douglas FL, Geiser R, Sowers JR. Metabolic and hemodynamic effects of magnesium supplementation in patients with essential hypertension. Am J Clin Nutr. 1990;51:665-9.

28. Denova-Gutiérrez E, Talavera JO, Huitrón-Bravo G, Méndez-Hernández P, Salmerón J. Sweetened beverage consumption and increased risk of metabolic syndrome in Mexican adults. Public Health Nutr. 2010;13:835-42.

29. Denova-Gutiérrez E, Castañón S, Talavera JO, Flores M, Macías N, RodríguezRamírez $\mathrm{S}$, et al. Dietary patterns are associated with different indexes of adiposity and obesity in an urban Mexican population. J Nutr. 2011;141:921-7.

30. Rosner B. Percentage points for a generalized ESD many-outlier procedure Technometrics. 1983;25:165-72

31. Hernández-Avila M, Romieu I, Parra S, Hernández-Avila J, Madrigal H, Willett W. Validity and reproducibility of a food frequency questionnaire to assess dietary intake of women living in Mexico City. Salud Publica Mex. 1998:40:133-40.

32. INSP. Bases de Datos del Valor Nutritivo de los Alimentos. Morelos: Compilación del Instituto Nacional de Salud Pública; 2004.

33. Willet WC. Nutritional Epidemiology. 2nd ed. New York: Oxford University Press; 1998.

34. Zeger SL, Liang KY. Longitudinal data analysis for discrete and continuous outcomes. Biometrics. 1986:42:121-30. 
35. Ascherio A, Hennekens C, Willett WC, Sacks F, Rosner B, Manson J, et al. Prospective study of nutritional factors, blood pressure, and hypertension among US women. Hypertension. 1996;27:1065-72.

36. Ascherio A, Rimm EB, Giovannucci EL, Colditz GA, Rosner B, Willett WC, et al. Prospective study of nutritional factors and hypertension among US men. Circulation. 1992;86:1475-84.

37. Zhao L, Stamler J, Yan LL, Zhou B, Wu Y, Liu K, et al. Blood pressure differences between northern and southern Chinese: role of dietary factors: The International Study on Macronutrients and Blood Pressure. Hypertension. 2004:43:1332-7.

38. Kass L, Weekes J, Carpenter L. Effect of magnesium supplementation on blood pressure: a meta-analysis. Eur J Clin Nutr. 2012;4:569-72.

doi:10.1186/2055-0928-1-6

Cite this article as: Huitrón-Bravo et al:: Dietary magnesium intake and risk of hypertension in a Mexican adult population: a cohort study. BMC Nutrition 2015 1:6.

\section{Submit your next manuscript to BioMed Central and take full advantage of:}

- Convenient online submission

- Thorough peer review

- No space constraints or color figure charges

- Immediate publication on acceptance

- Inclusion in PubMed, CAS, Scopus and Google Scholar

- Research which is freely available for redistribution 